\section{Tendências da epidemia de AIDS entre subgrupos sob maior risco no Brasil, 1980-2004}

\author{
Trends in the AIDS epidemic in groups at highest \\ risk in Brazil, 1980-2004
}

\author{
${ }_{1}^{1}$ Programa Nacional de DST \\ e AIDS, Ministério da Saúde, \\ Brasília, Brasil. \\ 2 Instituto de Comunicação \\ e Informação Científica \\ e Tecnológica em Saúde, \\ Fundação Oswaldo Cruz, \\ Rio de Janeiro, Brasil. \\ Correspondência \\ A. Barbosa Júnior \\ Programa Nacional de DST e \\ AIDS, Ministério da Saúde. \\ SEPN 511, Bloco C, 1o andar \\ Brasília, DF \\ 70750-543, Brasil. \\ aristides@aids.gov.br
}

\section{Abstract}

The objective of this paper was to present the trends in the AIDS epidemic in the population groups at highest risk in Brazil. Discriminant analysis was used to reclassify cases with unknown risk into one of the three groups: IDU (injecting drug users), MSM (men who have sex with men), and heterosexuals. AIDS incidence rates by gender and exposure category were estimated for the period 1980-2004. In 1980-1988,63.6\% of AIDS cases were homosexual or bisexual males and 10\% were females. Since 1988, there has been a decrease in the proportion of MSM and an increase in the other categories. Despite the incidence trends observed by exposure category, when the incidence rates were compared, the risk was much higher among MSM as compared to heterosexuals. Analysis of the AIDS epidemic dynamics in Brazil emphasizes the importance of MSM and male IDU as higher-risk groups.

Acquired Immunodeficiency Syndrome; Incidence; Risk Groups; Population Groups
Aristides Barbosa Júnior 1 Célia Landmann Szwarcwald 2 Ana Roberta Pati Pascom 1 Paulo Borges de Souza Júnior 2

\section{Introdução}

Muitos modelos têm sido desenvolvidos para entender a dinâmica da epidemia de AIDS e as inter-relações entre os determinantes da disseminação do HIV 1 .

Os modelos matemáticos têm destacado a contribuição desproporcional dos subgrupos populacionais sob maior risco na disseminação de doenças sexualmente transmissíveis (DST), tais como os usuários de drogas injetáveis (UDI), os homens que fazem sexo com outros homens (HSH) e as mulheres profissionais do sexo ${ }^{2}$. Por meio de modelagem matemática, mostra-se que para epidemias com uma baixa reprodução potencial, intervenções modestas em grupos de alto risco podem reduzir significativamente a incidência e prevalência de HIV. Já em países com epidemias generalizadas, intervenções efetivas devem incluir grupos de alto e baixo riscos já que as intervenções em grupos de alto risco são necessárias, mas não suficientes para obter rápido impacto e em larga escala ${ }^{3}$.

A Organização Mundial da Saúde (OMS) desenvolveu um modelo simples para estimar a taxa de prevalência de HIV, no qual os grupos sob maior risco têm papel destacado. Aperfeiçoado, posteriormente, pela UNAIDS (Programa Conjunto das Nações Unidas sobre HIV/AIDS), o modelo para países com epidemias concentradas consiste em estimar o tamanho das populações sob maior risco à infecção pelo HIV, estabele- 
cidas pelo próprio país, bem como as taxas de prevalência de HIV nestas populações. O número total de infectados é estimado pela soma dos números de infectados estimados em cada subgrupo sob maior risco 4,5 .

O modelo da UNAIDS destaca a importância dos subgrupos sob maior risco na dinâmica de disseminação da infecção pelo HIV, já que pequenas variações nas taxas de prevalência estimadas para os subgrupos sob maior risco podem provocar grandes alterações no número total de infectados pelo HIV, gerando até limitações no método proposto para estimar a prevalência de HIV na população geral 6 .

Outros grupos populacionais que têm sido considerados como elementos-chave na disseminação da infecção do HIV são aqueles que servem como ponte entre a população geral e os subgrupos populacionais sob maior risco, tais como os clientes de profissionais do sexo, parceiros de UDI, ou mulheres parceiras de homens bissexuais 7,8. Por exemplo, os clientes de profissionais do sexo que se envolvem em sexo desprotegido com um grupo de elevada prevalência do HIV representam uma "ponte" para transmissão do HIV entre este grupo e suas parceiras fixas 9 .

Sob a ótica dos modelos matemáticos, sabese que os fatores que dinamizam a disseminação do HIV por contato sexual são: a taxa de troca de parceiros sexuais; o tipo e a freqüência de contato sexual; e os padrões de relacionamento sexual entre subgrupos populacionais ${ }^{10}$. Sendo assim, o monitoramento do comportamento sexual tanto entre as populações sob maior risco como entre as "populações ponte" tem sido reconhecido como importante instrumento para o controle da disseminação do HIV. O conhecimento dos fatores envolvidos na transmissão do HIV, bem como a melhor compreensão sobre a dinâmica social de transmissão, são essenciais para subsidiar as medidas preventivas e garantir a efetividade das intervenções no nível de saúde coletiva 11 .

A epidemia de AIDS, no Brasil, teve início nos primeiros anos da década de 80 . Nesses mais de vinte anos, mostrou-se como uma epidemia "concentrada", mantendo uma taxa de prevalência da infecção pelo HIV na população geral em níveis menores do que $1 \% 12$, e níveis altos nos subgrupos populacionais sob maior risco à infecção pelo HIV, como os HSH e os UDI, que foram os mais afetados e infectados precocemente, no início da epidemia 13.

O objetivo do presente trabalho é apresentar as tendências das taxas de incidência de AIDS entre os UDI e heterossexuais masculinos, os $\mathrm{HSH}$ e as mulheres, por meio das informações disponíveis no Sistema Nacional de Agravos de Notificação (SINAN) e das estimativas do tama- nho destes subgrupos populacionais, realizadas a partir de inquérito de base populacional conduzido no país em 2004.

\section{Metodologia}

Tamanho das populações sob maior risco

No ano de 2004, realizou-se a Pesquisa de Conhecimento, Atitudes e Práticas (PCAP-BR, 2004), inquérito de base populacional para investigação do conhecimento, práticas e comportamentos de risco relacionados à infecção pelo HIV e outras doenças sexualmente transmissíveis na população brasileira de 15 a 54 anos. A execução da pesquisa significou a oportunidade de suprir informações para estimar o tamanho das populações sob maior risco no Brasil 14 .

O tamanho da amostra foi de 6 mil indivíduos de 15 a 54 anos de idade. A amostragem foi estratificada por macrorregião geográfica, tendo sido realizadas 900 entrevistas nas regiões Norte, Sul e Centro-Oeste, 1.100 na Região Nordeste, e 2.200 na Região Sudeste. Em cada uma das grandes regiões, a amostra foi realizada em múltiplos estágios: estados, setores censitários, e domicílios. Em cada estado, os setores foram selecionados com amostragem sistemática, com probabilidade proporcional ao tamanho. Em cada setor censitário foram selecionados sete domicílios. Em cada domicílio, apenas um morador respondeu ao questionário individual.

O questionário foi modular contendo as seguintes seções: condições sócio-econômicas; conhecimento sobre transmissão do HIV e outras DST; prevenção e controle de DST; testagem para o HIV; uso de drogas lícitas e ilícitas; práticas sexuais. Considerando que algumas das questões e temas abordados poderiam causar constrangimento, recusa ou falseamento nas informações, os módulos relativos ao uso de drogas e às práticas sexuais foram autopreenchidos, para melhorar a integridade das respostas. A seção de autopreenchimento utilizou folha à parte, que foi depositada, diretamente, em uma urna, como forma de garantia de sigilo ao entrevistado.

O projeto foi submetido ao Comitê de Ética em Pesquisa da Fundação Oswaldo Cruz (protocolo $n^{\circ}$. 243/04).

Com os dados coletados, foi possível estimar os tamanhos relativos dos seguintes subgrupos sob maior risco ao HIV: (a) HSH; (b) UDI; (c) mulheres profissionais do sexo; e (d) clientes de profissionais do sexo. As respostas foram obtidas na parte autopreenchida do questionário.

Especificamente para o presente trabalho, foram utilizadas as estimativas dos tamanhos 
dos grupos HSH e UDI. Para obter informações sobre a orientação sexual, a pergunta utilizada no questionário foi: "Normalmente, você tem relação sexual: somente com homens; somente com mulheres; mais com homens, mas às vezes com mulheres; mais com mulheres, mas às vezes com homens". No que se refere ao uso de drogas injetáveis, foi utilizada a seguinte pergunta: "Em relação à cocaína injetada na veia, você: nunca tomou; já experimentou, mas não usa mais; usa de vez em quando; usa freqüentemente".

\section{Incidência de AIDS}

Por meio das informações do SINAN relativas à AIDS, foram estabelecidas as incidências de AIDS entre os HSH, UDI do sexo masculino, heterossexuais masculinos e mulheres de 15 a 49 anos de idade.

Para a análise dos casos de AIDS notificados ao SINAN, foram retirados, primeiramente, os casos duplicados, identificados pela duplicação do nome, sexo, data de nascimento e Unidade da Federação de residência 15. Foram analisados 357.952 casos notificados ao SINAN até junho de 2007.

Os casos de 15 a 49 anos de idade foram avaliados por sexo e período de diagnóstico (19801988, 1989-1992, 1993-1996, 1997-2000, 20012004). Os casos masculinos foram agrupados por categoria de exposição: HSH; heterossexuais; UDI; categoria de exposição ignorada; outros.

Devido ao grande percentual de casos do sexo masculino com categoria de exposição ignorada, foi realizada uma análise discriminante, utilizando-se o aplicativo estatístico SPSS 13.0 (SPSS Inc., Chicago, Estados Unidos) para reclassificá-los em um dos três grupos: HSH, UDI e heterossexuais. O procedimento estatístico de análise discriminante consiste em construir um modelo de classificação baseado nas características dos casos para os quais se conhece a categoria de exposição, e que depois é aplicado aos casos com categoria de exposição ignorada para classificá-los em um dos três grupos. São geradas duas funções discriminantes, combinações lineares das variáveis independentes que fornecem a melhor discriminação entre os grupos 16. Foram utilizadas as seguintes variáveis independentes: (a) anos de estudo (0-3; 4-7; 8-11; 12+; ignorado); (b) idade (15-24; 25 29; 30-39; 40-49); (c) múltiplas parcerias (sim; não; ignorado); (d) parceiro tem múltiplas parcerias (sim; não; ignorado); (e) parceiro é UDI (sim; não; ignorado); (f) parceiro tem relações sexuais somente com homens (sim; não; ignorado); (g) parceiro tem relações sexuais somente com mulheres (sim; não; ignorado); (h) parceiro tem relações sexuais com homens e mulheres (sim; não; ignorado); (i) período de diagnóstico (1980-1988; 1989-1992; 1993-1996; 1997-2000; 2001-2004); (j) tamanho da população do município de residência (1-50.000; 50.001-200.000; 200.001-500.000; 500.001+); (l) presença de sintomas: sarcoma de Kaposi, tuberculose (pulmonar ou disseminada), tosse; (m) critério de definição de caso (Caracas; CDC; óbito); e (n) sobrevida ( $<5$ meses; 5 meses + ).

Alternativamente, todos aqueles com categoria de exposição ignorada foram considerados heterossexuais. Observa-se que essa alternativa de reclassificação dos casos com categoria de exposição ignorada fornece a estimativa máxima da taxa de incidência de AIDS entre os heterossexuais masculinos e é justificada pelo objetivo principal do trabalho de comparação das taxas de incidência de AIDS nos grupos sob maior risco (HSH e UDI).

Após a reclassificação dos casos com categoria de exposição ignorada, para cada uma das desagregações dos dados (sexo feminino; HSH; homens UDI, homens heterossexuais) foi realizado ajuste para o atraso da notificação após a data de diagnóstico para os anos de 2003 e 2004. Para tal, estima-se o percentual de casos notificados com menos de um ano após a data de diagnóstico, para o período 1988-1992, e para cada uma das desagregações sob estudo, sob a suposição de que todos os casos com diagnóstico nesse período já foram notificados ao SINAN.

Matematicamente, o percentual de casos notificados dentro de um ano após o diagnóstico é calculado por:

$$
P_{-} 1 a=\frac{N C \_1 a}{N C \_ \text {total }}
$$

Em que:

NC_la = número de casos diagnosticados no período 1988-1992 e notificados com menos de um ano após a data de diagnóstico;

NC_total = número total de casos diagnosticados no período 1988-1992 constantes no SINAN.

O número ajustado de casos nos anos 2003 e 2004 é calculado, então, pelo número de casos diagnosticados e notificados com menos de um ano após a data de diagnóstico, em cada ano, multiplicado pelo fator de correção, dado pelo inverso de P_la. Matematicamente, o ajuste é realizado da seguinte forma:

$N C_{\mathrm{i}}^{\prime}=N C_{\mathrm{i} \_} 1 a / P_{-} 1 a$

Para:

$\mathrm{i}=2003$, 2004, em que:

$\mathrm{NC}_{\mathrm{i}}^{\prime}=$ número total de casos no ano i corrigido pelo atraso na notificação; 
$\mathrm{NC}_{\mathrm{i}-} 1 \mathrm{a}=$ número de casos diagnosticados no ano i e notificados com menos de um ano após a data de diagnóstico.

Para o cálculo das taxas de incidência entre os HSH e os UDI masculinos foram utilizadas as estimativas dos tamanhos relativos destas populações, obtidas por meio da PCAP-BR, 2004, e as projeções anuais das populações de 15 a 49 anos no período 1985-2004, por sexo.

\section{Resultados}

\section{Tamanho dos subgrupos sob maior risco}

Na Tabela 1, são apresentadas as estimativas dos tamanhos relativos e absolutos das populações sob maior risco. O percentual de $\mathrm{HSH}$ entre os homens de 15 a 49 anos de idade foi de $3,2 \%$, correspondendo a cerca de um milhão e quinhentos mil homens, no Brasil, em 2004. Entre os homens sexualmente ativos nos últimos 12 meses, o percentual estimado foi de $3,5 \%$.

No que se refere aos UDI, o tamanho relativo foi de $0,2 \%$ de pessoas que fazem uso atual de drogas injetáveis na população brasileira de 15 a 49 anos de idade, correspondendo a, aproximadamente, 200 mil usuários, em 2004. O percentual foi maior para o sexo masculino, atingindo $0,3 \%$. Com relação ao uso de drogas injetáveis pelo menos uma vez na vida, as proporções foram de $0,9 \%$ (ambos os sexos), 1,3\% (sexo masculino) e $0,5 \%$ (sexo feminino).

\section{Incidência de AIDS por categoria de exposição}

Na Tabela 2, apresentam-se as incidências de AIDS por período de diagnóstico, sexo e categoria de exposição entre os casos masculinos. Observa-se uma grande proporção de casos do sexo masculino classificados com categoria de exposição ignorada, em torno de $12 \%$. Verificase, igualmente, um decréscimo do número total de casos no período 2001-2004 em relação ao período anterior, provavelmente devido ao atraso na notificação.

Mediante o procedimento estatístico de análise discriminante foi possível classificar os casos masculinos com categoria de exposição ignorada. Entre os casos com categoria de exposição conhecida, o percentual de acerto da classificação nos três grupos foi de $59,7 \%$ (58,1\% entre HSH; $64,3 \%$ entre heterossexuais; $56,4 \%$ entre UDI). Ter parceiro UDI, períodos de diagnóstico 1989-1992 e 1993-1996, parceiro não tem múltiplas parcerias, ter tuberculose, ser definido como caso pelo critério CDC foram as principais variáveis associadas à categoria UDI. Adicionalmente, sobrevida menor do que 5 meses, idade 15-24 anos, escolaridade baixa (0-4 anos de estudo), e morar em cidades de 50 a 200 mil habitantes foram também relacionadas à categoria UDI, embora não tão significativas. Parceiro tem relações com homens e mulheres, escolaridade alta (ensino médio completo ou superior), períodos de diagnóstico 1980-1988 e 1989-1992, ter sarcoma de Kaposi, residir em municípios com 500 mil habitantes ou mais e grupos etários 30-39, 40-49

Tabela 1

Tamanhos estimados (absoluto e relativo) das populações sob maior risco em relação à população total de 15-49 anos de idade. Brasil, 2004.

\begin{tabular}{|c|c|c|}
\hline \multirow[t]{2}{*}{ Populações sob maior risco } & \multicolumn{2}{|c|}{ População de 15-49 anos } \\
\hline & Tamanho relativo (\%) & Tamanho absoluto \\
\hline $\mathrm{HSH}$ & 3,2 & 1.538 .621 \\
\hline \multicolumn{3}{|l|}{ UDI } \\
\hline \multicolumn{3}{|l|}{ Pelo menos uma vez } \\
\hline Masculino & 1,3 & 625.065 \\
\hline Feminino & 0,5 & 247.916 \\
\hline Total & 0,9 & 878.986 \\
\hline \multicolumn{3}{|l|}{ Uso atual } \\
\hline Masculino & 0,3 & 144.246 \\
\hline Feminino & 0,2 & 99.166 \\
\hline Total & 0,2 & 195.330 \\
\hline
\end{tabular}

HSH: homens que fazem sexo com homens; UDI: usuários de drogas injetáveis. 
Incidência de AIDS e percentual do total de casos por sexo, período de diagnóstico e categoria de exposição. Brasil, 1980-2004.

\begin{tabular}{|c|c|c|c|c|c|c|}
\hline \multirow{2}{*}{$\begin{array}{l}\text { Categoria de } \\
\text { exposição }\end{array}$} & \multicolumn{5}{|c|}{ Período de diagnóstico } & \multirow[t]{2}{*}{ Total } \\
\hline & $1980-1988$ & 1989-1992 & 1993-1996 & $1997-2000$ & 2001-2004 & \\
\hline \multicolumn{7}{|c|}{ Sexo masculino } \\
\hline \multicolumn{7}{|l|}{$\mathrm{HSH}$} \\
\hline$n$ & 4.570 & 12.684 & 16.427 & 19.151 & 17.303 & 70.135 \\
\hline$\%$ & 54,6 & 33,0 & 22,3 & 19,3 & 17,6 & 22,1 \\
\hline \multicolumn{7}{|c|}{ Heterossexual } \\
\hline n & 513 & 3.845 & 10.965 & 19.683 & 23.366 & 58.372 \\
\hline$\%$ & 6,1 & 10,0 & 14,9 & 19,8 & 23,8 & 18,4 \\
\hline \multicolumn{7}{|l|}{ UDI } \\
\hline $\mathrm{n}$ & 1.321 & 10.206 & 15.432 & 14.013 & 8.517 & 49.489 \\
\hline$\%$ & 15,8 & 26,6 & 21,0 & 14,1 & 8,7 & 15,6 \\
\hline \multicolumn{7}{|l|}{ Outra } \\
\hline $\mathrm{n}$ & 276 & 596 & 612 & 229 & 150 & 1.863 \\
\hline$\%$ & 3,3 & 1,6 & 0,8 & 0,2 & 0,2 & 0,6 \\
\hline \multicolumn{7}{|l|}{ Ignorada } \\
\hline $\mathrm{n}$ & 850 & 4.581 & 11.574 & 12.160 & 10.820 & 39.985 \\
\hline$\%$ & 10,2 & 11,9 & 15,7 & 12,2 & 11,0 & 12,6 \\
\hline \multicolumn{7}{|l|}{ Sexo feminino } \\
\hline $\mathrm{n}$ & 839 & 6.490 & 18.605 & 34.201 & 37.976 & 98.111 \\
\hline$\%$ & 10,0 & 16,9 & 25,3 & 34,4 & 38,7 & 30,9 \\
\hline \multicolumn{7}{|l|}{ Total } \\
\hline$N$ & 8.369 & 38.402 & 73.615 & 99.437 & 98.132 & 317.955 \\
\hline$\%$ & 100,0 & 100,0 & 100,0 & 100,0 & 100,0 & 100,0 \\
\hline
\end{tabular}

HSH: homens que fazem sexo com homens; UDI: usuários de drogas injetáveis.

foram as variáveis que melhor discriminaram a categoria HSH. Múltiplas parcerias, parceiro só tem parceiros homens e morar em cidades pequenas (1-50 mil habitantes) foram as variáveis mais importantes para classificação na categoria de heterossexuais.

Os dados dispostos na Tabela 3 mostram a dinâmica da epidemia de AIDS no Brasil. Considerando a reclassificação dos casos pelo procedimento de análise discriminante, no período 1980-1988, os casos homossexuais ou bissexuais masculinos correspondiam a $63,6 \%$ da totalidade dos casos, enquanto os casos UDI a $16,5 \%$. A proporção de mulheres era de apenas $10 \%$. No período posterior (1989-1992), há um decréscimo importante no papel desempenhado pelos $\mathrm{HSH}$, que passam a representar $38,1 \%$ da totalidade dos casos. Paralelamente, ocorre um acréscimo nas outras categorias de exposição, principalmente entre UDI. No terceiro período de tempo analisado (1993-1996), a tendência de decréscimo permanece entre os HSH enquanto os casos heterossexuais masculinos e as mulhe- res mostram um aumento pronunciado. Já a proporção de casos UDI mostra inversão na tendência, passando de $32,6 \%$ a $28,5 \%$ do segundo para o terceiro período de tempo. No último período analisado (2001-2004), as mulheres representam $38,7 \%$ dos casos de AIDS, os homens heterossexuais, 33,3\%, enquanto os HSH e UDI, $18,3 \%$ e $9,5 \%$, respectivamente.

As tendências da incidência de AIDS no período 1985-2004 podem ser melhor visualizadas na Figura 1. Enquanto as mulheres e os heterossexuais masculinos mostram as maiores incidências e tendências nitidamente crescentes no período todo, os HSH mostram crescimento até 1998 e depois ligeiro declínio. Já os UDI atingem incidência máxima em 1995, para decrescer em ritmo acelerado após esse ano.

Embora as tendências temporais das taxas de incidência de AIDS obedeçam ao mesmo padrão de evolução das incidências por categoria de exposição, a análise das taxas de incidência, porém, mostra quadro bem diferente (Figura 2): sobressai o risco entre os HSH e UDI quando 
Incidência de AIDS e percentual do total de casos por sexo, período de diagnóstico e categoria de exposição após reclassificação dos casos com categoria de exposição ignorada pela análise discriminante. Brasil, 1980-2004.

\begin{tabular}{|c|c|c|c|c|c|c|}
\hline \multirow{2}{*}{$\begin{array}{l}\text { Categoria de } \\
\text { exposição }\end{array}$} & \multicolumn{5}{|c|}{ Período de diagnóstico } & \multirow[t]{2}{*}{ Total } \\
\hline & 1980-1988 & 1989-1992 & 1993-1996 & $1997-2000$ & 2001-2004 & \\
\hline \multicolumn{7}{|c|}{ Sexo masculino } \\
\hline \multicolumn{7}{|l|}{$\mathrm{HSH}$} \\
\hline$n$ & 5.319 & 14.634 & 20.459 & 21.679 & 18.004 & 80.095 \\
\hline$\%$ & 63,6 & 38,1 & 27,8 & 21,8 & 18,3 & 25,2 \\
\hline \multicolumn{7}{|c|}{ Heterossexual } \\
\hline$n$ & 555 & 4.162 & 12.956 & 24.267 & 32.636 & 74.576 \\
\hline$\%$ & 6,6 & 10,8 & 17,6 & 24,4 & 33,3 & 23,5 \\
\hline \multicolumn{7}{|l|}{ UDI } \\
\hline n & 1.380 & 12.520 & 20.983 & 19.061 & 9.366 & 63.310 \\
\hline$\%$ & 16,5 & 32,6 & 28,5 & 19,2 & 9,5 & 19,9 \\
\hline \multicolumn{7}{|l|}{ Outra } \\
\hline $\mathrm{n}$ & 276 & 596 & 612 & 229 & 150 & 1863 \\
\hline$\%$ & 3,3 & 1,6 & 0,8 & 0,2 & 0,2 & 0,6 \\
\hline \multicolumn{7}{|l|}{ Sexo feminino } \\
\hline $\mathrm{n}$ & 839 & 6.490 & 18.605 & 34.201 & 37.976 & 98.111 \\
\hline$\%$ & 10,0 & 16,9 & 25,3 & 34,4 & 38,7 & 30,9 \\
\hline \multicolumn{7}{|l|}{ Total } \\
\hline $\mathrm{N}$ & 8.369 & 38.402 & 73.615 & 99.437 & 98.132 & 317.955 \\
\hline$\%$ & 100,0 & 100,0 & 100,0 & 100,0 & 100,0 & 100,0 \\
\hline
\end{tabular}

HSH: homens que fazem sexo com homens; UDI: usuários de drogas injetáveis.

comparado aos encontrados entre os homens heterossexuais e as mulheres. Em 1995, a taxa de incidência entre UDI do sexo masculino está na faixa de 8,7 por mil usuários, enquanto a taxa de heterossexuais masculinos é de 0,08 por mil, aproximadamente 115 vezes menor. Nesse mesmo ano, a taxa de incidência de AIDS entre HSH é de 3,3 por mil, 44 vezes maior que a taxa entre homens heterossexuais.

Apesar das tendências inversas no tempo, isto é, decréscimo para as incidências de casos HSH e UDI e acréscimo entre os heterossexuais masculinos e as mulheres, quando as taxas de incidência são comparadas, o risco é pronunciadamente maior entre os primeiros. Em 2004, a maior taxa ainda é encontrada entre homens UDI, aproximadamente 15 vezes maior que a taxa entre heterossexuais masculinos (Figura 2).

Ainda que todos os casos masculinos com categoria de exposição ignorada sejam considerados heterossexuais masculinos, o mesmo padrão de evolução temporal das taxas de incidência é encontrado (Figuras 3 e 4). Os UDI apresentam a maior taxa em todo o período analisado, alcançando o mesmo patamar que os HSH somente em 2004. Para esse ano, as taxas de incidência de
AIDS em ambos os grupos permanecem bem superiores à encontrada entre os homens heterossexuais, com valores aproximadamente 13 vezes maiores.

\section{Discussão}

A análise da dinâmica da epidemia de AIDS no Brasil mostra, sem dúvida, a importância dos grupos HSH e UDI enquanto grupos de risco diferenciado.

Os UDI do sexo masculino mostraram o maior risco, com taxas no começo da epidemia em torno de 200 vezes maiores do que a taxa entre os heterossexuais. Esse grupo foi o que mostrou, por outro lado, a maior velocidade de decréscimo, embora ainda seja o que detém o maior risco. Esse decréscimo pode estar relacionado a três fatores: à política de redução de danos adotada no Brasil, com aumento de práticas de uso seguro de drogas; à mudança no perfil de uso de drogas no país, com a migração do uso de drogas injetáveis para outras formas de uso ou para outras drogas; finalmente, ao esgotamento de suscetíveis 17,18. 


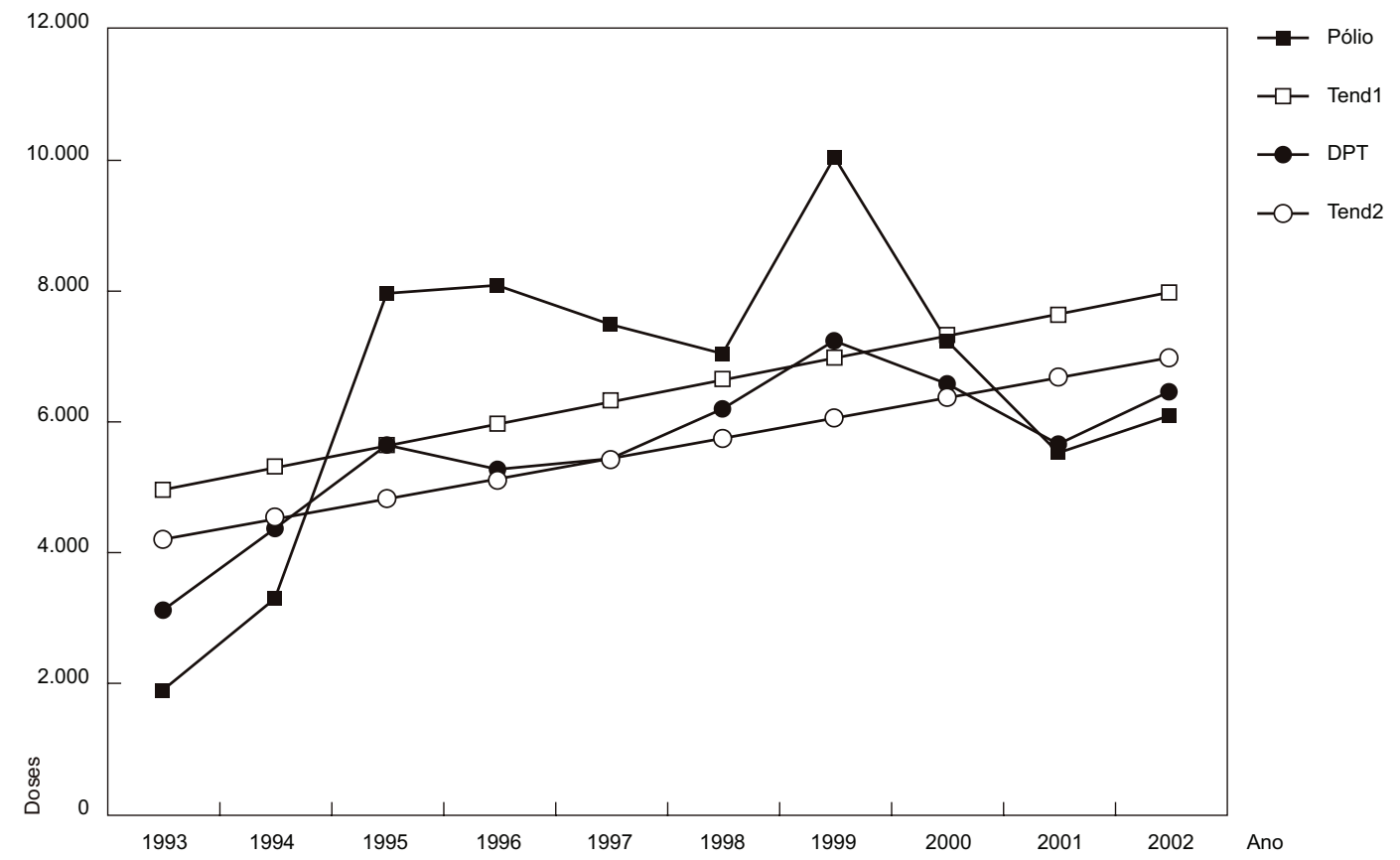

Fonte: Sistema de Informação de Agravos de Notificação (SINAN), até julho de 2007.

HSH: homens que fazem sexo com homens; UDI: usuários de drogas injetáveis.

* Corrigido pelo atraso de notificação.

No caso de HSH, observou-se acréscimo no primeiro período de tempo, e, posteriormente, tendência de ligeiro declínio, embora a taxa de incidência tenha permanecido bem mais elevada que a da população geral. Esse declínio pode estar relacionado à maior freqüência de práticas sexuais seguras entre os HSH quando comparadas à população masculina em geral 19.

Apesar do crescimento dos casos em mulheres e heterossexuais masculinos, a epidemia de AIDS no Brasil mantém-se concentrada, segundo os parâmetros da UNAIDS e da OMS ${ }^{20}$, com taxa de prevalência do HIV menor do que $1 \%$ em gestantes e maior do que $5 \%$ nos subgrupos considerados. A análise apresentada neste estudo aponta que a epidemia de AIDS no país deve manter-se como concentrada ainda por muitos anos, caso não haja nenhuma modificação significativa nos determinantes da epidemia.

Os resultados encontrados no presente trabalho enfatizam, pois, a importância do monitoramento das práticas de risco associadas à infecção pelo HIV nesses grupos populacionais sob maior risco, que não têm sido adequadamente acompanhados no Brasil como um todo, especialmente devido às dificuldades inerentes à realização de estudos com essas populações.

Na população geral, o conhecimento, atitudes e práticas relacionadas à infecção pelo HIV têm sido monitorados, periodicamente, por pesquisas de base populacional 21. No entanto, devido ao tamanho das populações sob maior risco, os métodos de amostragem tradicionais são ineficientes para obtenção de resultados específicos, gerando a necessidade de estudos especiais com métodos de amostragem apropriados para populações de difícil acesso 22 .

O SINAN apresenta algumas limitações na qualidade das suas informações que merecem ser consideradas nesta discussão. A primeira diz respeito à completitude do campo categoria de exposição entre homens. Em uma proporção significativa de casos, essa variável não está preenchida. Para contornar esse problema, foi 
Figura 2

Taxa de incidência de AIDS (por 100 mil) por sexo, ano de diagnóstico e categoria de exposição após reclassificação dos ignorados pela análise discriminante. Brasil, 1985-2004.

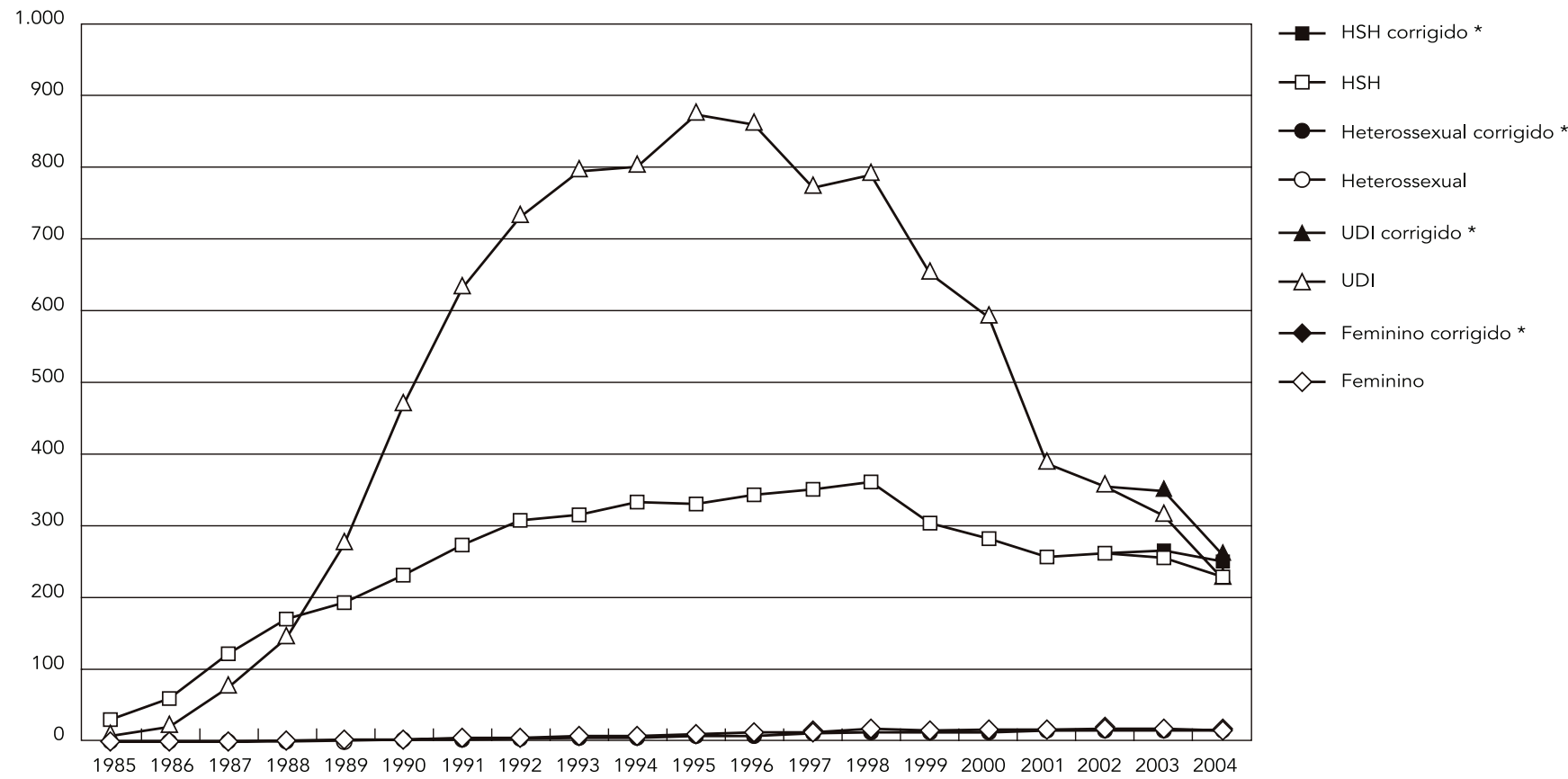

Fonte: Sistema de Informação de Agravos de Notificação (SINAN), até julho de 2007.

$\mathrm{HSH}$ : homens que fazem sexo com homens; UDI: usuários de drogas injetáveis.

* Corrigido pelo atraso de notificação.

realizado o procedimento estatístico de análise discriminante. Entretanto, na hipótese extrema de considerarmos que todos os casos com categoria de exposição ignorada fossem heterossexuais, os padrões de evolução temporal das taxas de incidência não seriam modificados substancialmente.

Uma segunda limitação do SINAN é que não é possível analisar a tendência dos casos entre mulheres profissionais do sexo a partir das suas informações. No sistema, são diferenciadas apenas as mulheres com múltiplas parcerias, sem padronização da definição de múltiplas parcerias. Também não há possibilidade de analisar os clientes de profissionais do sexo, já que não há registro específico desta informação no banco de dados.

Adicionalmente, coloca-se a dificuldade em estimar-se o tamanho dos subgrupos populacionais sob maior risco. É possível que o tamanho da população de HSH esteja subestimado, levando-se em consideração que a estimativa utilizada foi baseada em inquérito domiciliar. Estudos realizados em outros países mostram uma proporção de $\mathrm{HSH}$ variando entre $4 \%$ e $7 \%$ 23,24. No entanto, mesmo assumindo-se esse acréscimo no tamanho populacional desse grupo, as tendências observadas não se modificariam expressivamente. Da mesma forma, podese ter sobreestimado as taxas de incidência entre UDI, principalmente no início da epidemia, já que o tamanho relativo deste grupo utilizado para o cálculo dos denominadores foi baseado no estudo realizado em 2004, que foi mantido constante no período 1985-2004. A diminuição do tamanho da população UDI foi corroborada por Bastos et al. 25 em inquérito realizado em 2005. Sendo assim, as séries temporais devem ser examinadas à luz dessa limitação.

Em suma, mostrou-se com base nas estatísticas oficiais de notificação de AIDS que os grupos HSH e UDI masculino têm risco acrescido quando comparados aos homens heterossexuais. Reconhecendo a existência de situações 
Incidência de AIDS por sexo, ano de diagnóstico e categoria de exposição após reclassificar os ignorados como heterossexuais masculinos. Brasil, $1985-2004$.

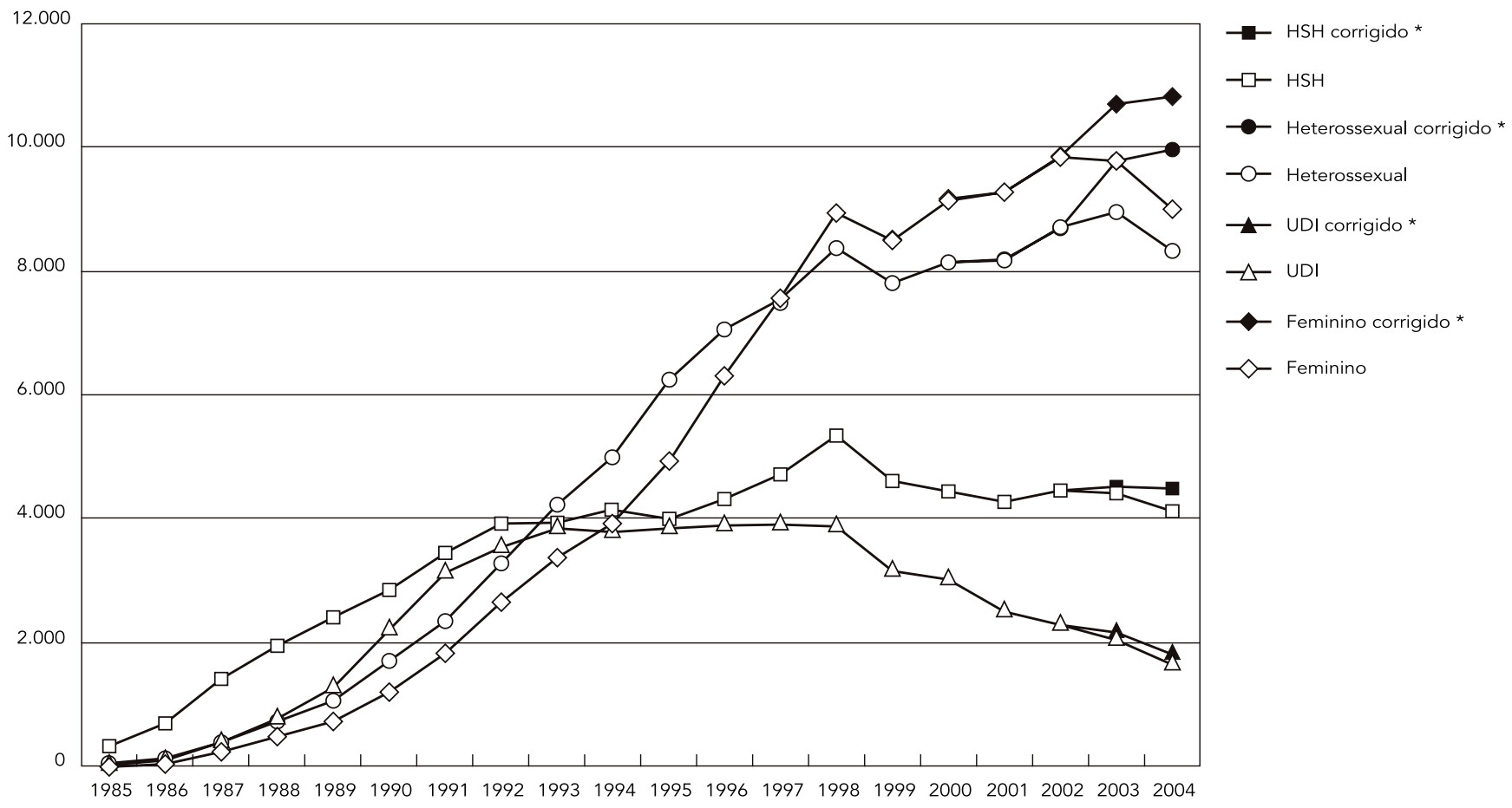

Fonte: Sistema de Informação de Agravos de Notificação (SINAN), até julho de 2007.

HSH: homens que fazem sexo com homens; UDI: usuários de drogas injetáveis.

* Corrigido pelo atraso de notificação.

específicas que favorecem a infecção pelo HIV e que continuam contribuindo para que certos grupos sejam mais suscetíveis, na fase atual de planejamento das ações, vários novos esforços têm sido dirigidos aos grupos populacionais sob maior risco. Incluem-se entre as estratégias, a amostragem em populações de difícil acesso para monitorar o comportamento de risco relacionado à infecção pelo HIV nesses subgrupos populacionais 21 . 
Figura 4

Taxa de incidência de AIDS (por 100 mil) por sexo, ano de diagnóstico e categoria de exposição após reclassificar os ignorados como heterossexuais masculinos. Brasil, 1985-2004.

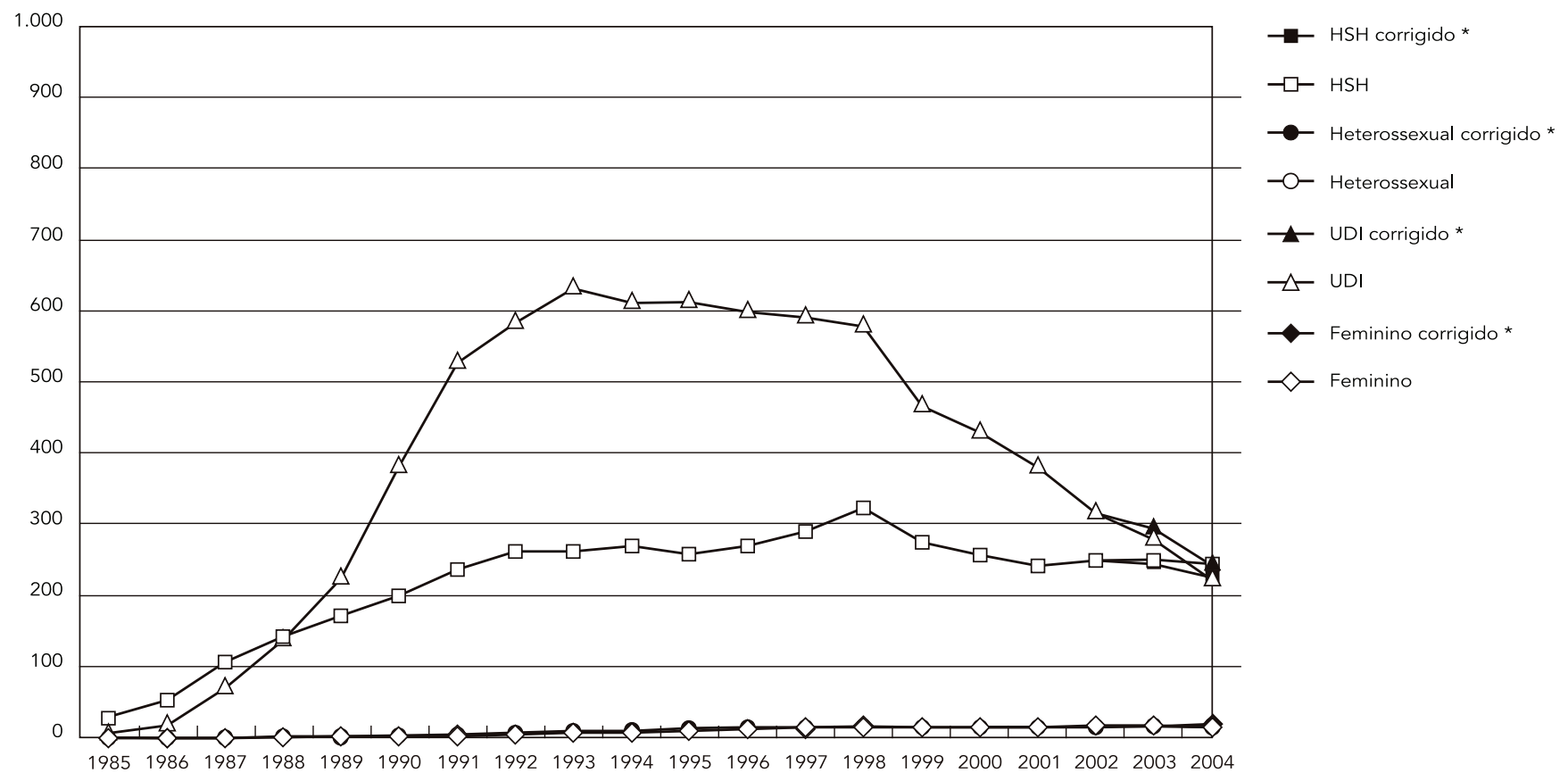

Fonte: Sistema de Informação de Agravos de Notificação (SINAN), até julho de 2007.

$\mathrm{HSH}$ : homens que fazem sexo com homens; UDI: usuários de drogas injetáveis.

* Corrigido pelo atraso de notificação.

\section{Resumo}

O presente trabalho tem por objetivo apresentar as tendências da epidemia de AIDS em grupos populacionais sob maior risco no Brasil. A técnica de análise discriminante foi utilizada para reclassificação dos casos masculinos com categoria de exposição ignorada em um dos três grupos homens que fazem sexo com homens (HSH), usuários de drogas injetáveis (UDI) ou heterossexuais. Foram estimadas as taxas de incidência de AIDS por sexo e categoria de exposição no período 1980-2004. No período 1980-1988, os casos homossexuais ou bissexuais masculinos correspondiam a 63,6\% dos casos, e a proporção de mulheres era de 10\%. Posteriormente, há um decréscimo importante no papel desempenhado pelos HSH e ocorre um acréscimo nas outras categorias de exposição. Apesar das tendências de decréscimo para as incidências de casos HSH e UDI e acréscimo entre os heterossexuais masculinos e as mulheres, quando as taxas de incidência são comparadas o risco é maior entre os HSH e UDI. A análise da dinâmica da epidemia de AIDS no Brasil mostra a importância dos grupos HSHe UDI masculinos enquanto grupos de risco diferenciado.

Síndrome de Imunodeficiência Adquirida; Incidência; Grupos de Risco; Grupos Populacionais

\section{Colaboradores}

A. Barbosa Júnior foi o principal mentor do trabalho e responsável pela elaboração do texto. C. L. Szwarcwald participou da elaboração do texto e da concepção do artigo. A. R. P. Pascom trabalhou na análise estatística de dados e participou da redação dos resultados e discussão. P. R. B. Souza Júnior trabalhou na metodologia e elaborou tabelas e gráficos. 


\section{Referências}

1. Chin J, Lwanga SK. Estimation and projection of adult AIDS cases: a simple epidemiological model. Bull World Health Organ 1991; 69:399-406.

2. Potts M, Anderson R, Boily MC. Slowing the spread of human immunodeficiency virus in developing countries. Lancet 1991; 338:608-13.

3. Boily MC, Lowndes C, Alary M. The impact of HIV epidemic phases on the effectiveness of core group interventions: insights from mathematical models. Sex Transm Infect 2002; 78 Suppl 1:78-90.

4. Walker N, Stanecki KA, Brown T, Stover J, Lazzari S, Garcia-Calleja JM, et al. Methods and procedures for estimating HIV/AIDS and its impact: the UNAIDS/WHO estimates for the end of 2001. AIDS 2003; 17:2215-25.

5. Ghys PD, Brown T, Grassly NC, Garnett G, Stanecki KA, Stover J, et al. The UNAIDS Estimation and Projection Package: a software package to estimate and project national HIV epidemics. Sex Transm Infect 2004; 80 Suppl 1:i5-9.

6. Brown T, Grassly NC, Garnett G, Stanecki K. Improving projections at the country level: the UNAIDS Estimation and Projection Package 2005. Sex Transm Infect 2006; 82 Suppl 3:iii34-40.

7. Lowndes CM, Alary M, Meda H, Gnintoungbe CA, Mukenge-Tshibaka L, Adjovi C, et al. Role of core and bridging groups in the transmission dynamics of HIV and STIs in Cotonou, Benin, West Africa. Sex Transm Infect 2002; 78 Suppl 1:i69-77.

8. Qian HZ, Vermund SH, Wang N. Risk of HIV/AIDS in China: subpopulations of special importance. Sex Transm Infect 2005; 81:442-7.

9. Morris M, Podhisita C, Wawer MJ, Handcock MS. Bridge populations in the spread of HIV/AIDS in Thailand. AIDS 1996; 10:1265-71.

10. Boily MC, Mâsse B. Mathematical models of disease transmission: a precious tool for the study of sexually transmitted diseases. Can J Public Health 1997; 88:255-65.

11. Joint United Nations Programme on HIV/AIDS. Report on the global HIV/AIDS epidemic. Geneva: Joint United Nations Programme on HIV/AIDS; 2000.

12. Szwarcwald CL, Souza Junior PRB. Estimativa de prevalência de HIV na população brasileira de 15-49 anos, 2004. Boletim Epidemiológico AIDST 2006; III:11-5.

13. Barcellos C, Bastos FI. Redes sociais e difusão da AIDS no Brasil. Bol Oficina Sanit Panam 1996; 121:11-24.

14. Szwarcwald CL, Barbosa-Júnior A, Pascom ARP, Souza-Júnior PR. Knowledge, practices and behaviours related to HIV transmission among the Brazilian population in the 15-54 years age group, 2004. AIDS 2005; 19 Suppl 4:S51-8.
15. Ministério da Saúde. Nota técnica 1 - metodologia de revisão da base de dados do sistema de notificação de casos de Aids - SINAN-Aids. Boletim Epidemiológico AIDST 2004; I:42-5.

16. Morrison, Donald F. Multivariate statistical methods. 3rd Ed. Columbus: McGraw-Hill; 1990. (McGraw-Hill Series in Probability and Statistics).

17. Fonseca EM, Nunn A, Souza Junior PB, Bastos FI, Ribeiro JM. Descentralização, AIDS e redução de danos: a implementação de políticas públicas no Rio de Janeiro, Brasil. Cad Saúde Pública 2007; 23:2134-44.

18. Caiaffa WT, Bastos FI, Freitas LL, Mingoti SA, Proietti FA, Carneiro-Proietti AB, et. al. The contribution of two Brazilian multi-center studies to the assessment of HIV and HCV infection and prevention strategies among injecting drug users: the AjUDE-Brasil I and II Projects. Cad Saúde Pública 2006; 22:771-82.

19. Coordenação Nacional de DST e Aids. PCAP - Pesquisa de Conhecimento, Atitudes e Práticas na População Brasileira 2004. Brasília: Ministério da Saúde; 2006.

20. Coordenação Nacional de DST e Aids. Vigilância do HIV no Brasil - novas diretrizes. Brasília: Ministério da Saúde; 2002. (Série Referência, 2).

21. Barbosa Junior A, Pascom AR, Szwarcwald CL, Dhalia CB, Monteiro L, Simão MB. Proposed UNGASS indicators and the monitoring of the AIDS epidemic in Brazil. Rev Saúde Pública 2006; 40 Suppl 1:94-100.

22. Magnani R, Sabin K, Saidel T, Heckathorn D. Review of sampling hard-to-reach and hidden populations for HIV surveillance. AIDS 2005; 19 Suppl 2: S67-72.

23. Lieb S, Trepka MJ, Thompson DR, Arons P, Liberti T, Maddox L, et al. Men who have sex with men: estimated population sizes and mortality rates by race/ethnicity, Miami-Dade County, Florida. J Acquir Immune Defic Syndr 2007; 46:485-90.

24. Ning Z, Pan QC, Zheng XH, Zhang W, Lv F, Kang LY. Study on using the multiplier method in estimating the size of men who have sex with men population in Shanghai. Zhonghua Liu Xing Bing Xue Za Zhi 2007; 28:848-50.

25. Bastos FI, Bongertz V, Teixeira SL, Morgado MG, Hacker MA. Is human immunodeficiency virus/ acquired immunodeficiency syndrome decreasing among Brazilian injection drug users? Recent findings and how to interpret them. Mem Inst Oswaldo Cruz 2005; 100:91-6.

Recebido em 12/Ago/2008

Versão final reapresentada em 09/Dez/2008 Aprovado em 19/Dez/2008 